\title{
Comparative study of the loop-mediated isothermal amplification method and the QIAGEN therascreen PCR kit for the detection of EGFR mutations in non-small cell lung cancer
}

\author{
Yuichi Saito ${ }^{1,2}$, Nobumasa Takahashi ${ }^{2}$, Atsuka Matsui ${ }^{3}$, Satoru Michiyuki ${ }^{3}$, Yoshikane Yamauchi ${ }^{1}$, \\ Yoshihiko Shimizu ${ }^{4}$, Eishin Hoshi ${ }^{2}$, Yukinori Sakao ${ }^{1}$, Masafumi Kawamura ${ }^{1}$ \\ ${ }^{1}$ Department of Surgery, Teikyo University School of Medicine, Tokyo, Japan; ${ }^{2}$ Department of Thoracic Surgery, Saitama Cardiovascular and \\ Respiratory Center, Saitama, Japan; ${ }^{3}$ Fundamental Research Laboratory, Fundamental Technology Research Department, Eiken Chemical Co., Ltd., \\ Tochigi, Japan; ${ }^{4}$ Department of Pathology, Saitama Cardiovascular and Respiratory Center, Saitama, Japan \\ Contributions: (I) Conception and design: Y Saito, N Takahashi, Y Shimizu, E Hoshi; (II) Administrative support: Y Sakao, M Kawamura; (III) \\ Provision of study materials or patients: N Takahashi, Y Yamauchi, Y Shimizu; (IV) Collection and assembly of data: Y Saito, N Takahashi, A Matsui, \\ S Michiyuki, Y Shimizu; (V) Data analysis and interpretation: Y Saito, N Takahashi; (VI) Manuscript writing: All authors; (VII) Final approval of \\ manuscript: All authors. \\ Correspondence to: Yuichi Saito. Department of Surgery, Teikyo University School of Medicine, 2-11-1 Kaga, Itabashi-ku, Tokyo 173-8605, Japan. \\ Email: k3699004@gmail.com.
}

Background: Epidermal growth factor receptor $(E G F R)$ mutations are important biomarkers in the treatment of patients with advanced or metastatic diseases. The therascreen EGFR Rotor-Gene Q (RGQ) PCR Kit ${ }^{\circledR}$ (Qiagen, Inc.) is an approved diagnostic test for EGFR mutations in non-small cell lung cancer (NSCLC). This study aims to investigate the diagnostic capability of a loop-mediated isothermal amplification (LAMP) assay as an accurate, efficient, and cost-effective alternative to the therascreen assay.

Methods: EGFR mutations were investigated by LAMP and therascreen assays using tissue samples that were surgically resected or biopsied from 117 consecutive patients with NSCLC tumors. The EGFR status from the LAMP assay was compared with that of the therascreen assay. Next-generation sequencing (NGS) was performed to confirm EGFR status of tumors that did not match in both assays. To establish an optimal LAMP AUC value, receiver operating characteristics (ROC) curve analysis was performed within tumors with exon 19 deletion or L858R point mutation.

Results: Of the 117 tumors assayed, 45 tumors with EGFR mutations and 68 tumors with EGFR wild type were matched in both assays, four tumors having mismatched EGFR statuses. NGS further confirmed that two of the four discordant tumors had the same EGFR status that was determined by the LAMP assay. The AUC values were 0.973 (95\% CI: 0.929-1.00) in exon 19 deletion, and 0.952 (95\% CI: 0.885-1.00) in L858R point mutation. In exon 19 deletion, sensitivity, specificity, and accuracy were $89.3 \%, 98.9 \%$, and $96.6 \%$, respectively, and 94.7\%, 95.9\%, and 95.7\%, respectively, in L858R using AUC value of 0.222.

Conclusions: The LAMP assay compared favorably with the therascreen assay and has potential as an effective, simple, rapid, and low-cost diagnostic alternative. Based on these results, a liquid biopsy LAMP system should be developed for point-of-care testing of oncogenes in the near future.

Keywords: Lung cancer; adenocarcinoma; epidermal growth factor receptor (EGFR); loop-mediated isothermal amplification (LAMP); polymerase chain reaction

Submitted Aug 10, 2020. Accepted for publication Nov 26, 2020.

doi: $10.21037 /$ jtd-20-2642

View this article at: http://dx.doi.org/10.21037/jtd-20-2642

\footnotetext{
^ ORCID: 0000-0002-2025-9226.
} 


\section{Introduction}

Lung cancer, accounting for 2.09 million new cases and 1.76 million deaths in the GLOBOCAN 2018 database (1) and 2.28 hundred thousand new cases and 1.42 hundred thousand deaths in the United States (2), is the most common cancer and the leading cause of death from cancer in many developed countries. However, overall survival and progression-free survival have improved for patients with advanced non-small cell lung cancer (NSCLC) who have specific predictive biomarkers and have received molecular targeted therapy or immunotherapy compared with those receiving cytotoxic chemotherapy (3-10). Currently, almost all guidelines for lung cancer strongly recommend the investigation of several established biomarkers (EGFR mutations, ALK and ROS1 rearrangement, BRAF V600E point mutations, and PD-L1 expression levels) prior to initial treatment of patients with advanced NSCLC (11-16).

NSCLC with EGFR mutations is highly responsive to EGFR tyrosine kinase inhibitors (TKIs), such as gefitinib (Iressa $^{\circledR}$; AstraZeneca UK Limited) (17). Gefitinib was approved for the first-line treatment of patients with metastatic NSCLC on July 5, 2002 in Japan (18), and was later approved for metastatic NSCLC patients on May 5, 2003 in the United States. Simultaneously, the therascreen EGFR Rotor-Gene Q (RGQ) PCR Kit ${ }^{\circledR}$ (therascreen assay; Qiagen, Inc.) was approved as a companion diagnostic test by the Ministry of Health, Labour and Welfare in Japan and by the United States Food and Drug Administration (FDA) $(19,20)$.

The therascreen EGFR RGQ PCR Kit ${ }^{\circledR}$ is a commercial real-time polymerase chain reaction (RT-PCR) test kit, which detects 21 EGFR mutations in exons 18, 19, 20, and 21 against a background of wild type genomic DNA. After purification of DNA extracted from formalin-fixed paraffinembedded (FFPE) tissue, aberrant DNA is amplified using amplification refractory mutation system (ARMS) primers with Rotor-Gene Q MDx ${ }^{\circledR}$ (Qiagen, Inc., Tokyo, Japan), which is a dedicated thermal cycler. While this assay can be performed within eight hours, it requires the use of sophisticated equipment and procedures, and specimens that are adequate sample size and of a high quality.

Recently, next-generation sequencing (NGS), which enables massively paralleled DNA sequencing, has been introduced into practical clinics in some developed countries (21-25). Since this technology is able to sequence millions of target genes simultaneously, many oncologists are expecting that the genotyping of lung cancer will be more cost- and time-effective. However, NGS has several limitations: (I) expensive equipment; (II) requires experienced technicians; (III) requires experienced biostatisticians; and (IV) it is time-consuming. NGS is particularly unsuited for patients with far advanced disease because EGFR status should be investigated as soon as possible to allow fast precision medicine and first-line therapy with EGFR-TKIs.

Loop-mediated isothermal amplification (LAMP) is an alternative PCR based technology with high levels of specificity and amplification efficiency under isothermal conditions (26-28). It is being used worldwide in bacteriology (29-32), protozoology (33), mycology (34), and virology including SARS-CoV-2 (35-41). This assay has the following advantages: (I) high speed detection within 30 minutes after the amplification reaction begins; (II) unpurified samples can be used directly; and (III) high sensitivity and specificity compared with conventional PCR methods. Furthermore, the cost-effectiveness of LAMP was proved in tuberculosis by World Health Organization because no expensive thermal cycler is needed (42). Although the LAMP assay has not be put into practice used in clinical oncology, it could provide more rapidly, simply, and inexpensively method for detecting oncogene.

In this study, we aimed to develop a new point-ofcare testing LAMP assay for detecting EGFR mutations in oncology. To evaluate the sensitivity and specificity, we compared results between the LAMP assay and the therascreen assay. Additionally, by analyzing the area under the receiver operator characteristic curve (ROC), we calculated the optimal threshold that distinguishes between EGFR mutations (exon 19 deletion or L858R) and the wild type. We present the following article in accordance with the STARD reporting checklist (available at http://dx.doi. org/10.21037/jtd-20-2642).

\section{Methods}

\section{Study design}

This study was a prospective study, without intervention, that was designed to elucidate the sensitivity and specificity of the LAMP assay compared with the therascreen assay. Clinical data and specimens were collected from consecutive resected tumor tissues from patients at Saitama Cardiovascular and Respiratory Center (Saitama, Japan). The present study was approved by The Institutional Review Board of the Saitama Cardiovascular and Respiratory Center (approval No. 2018032 and 2019038). 


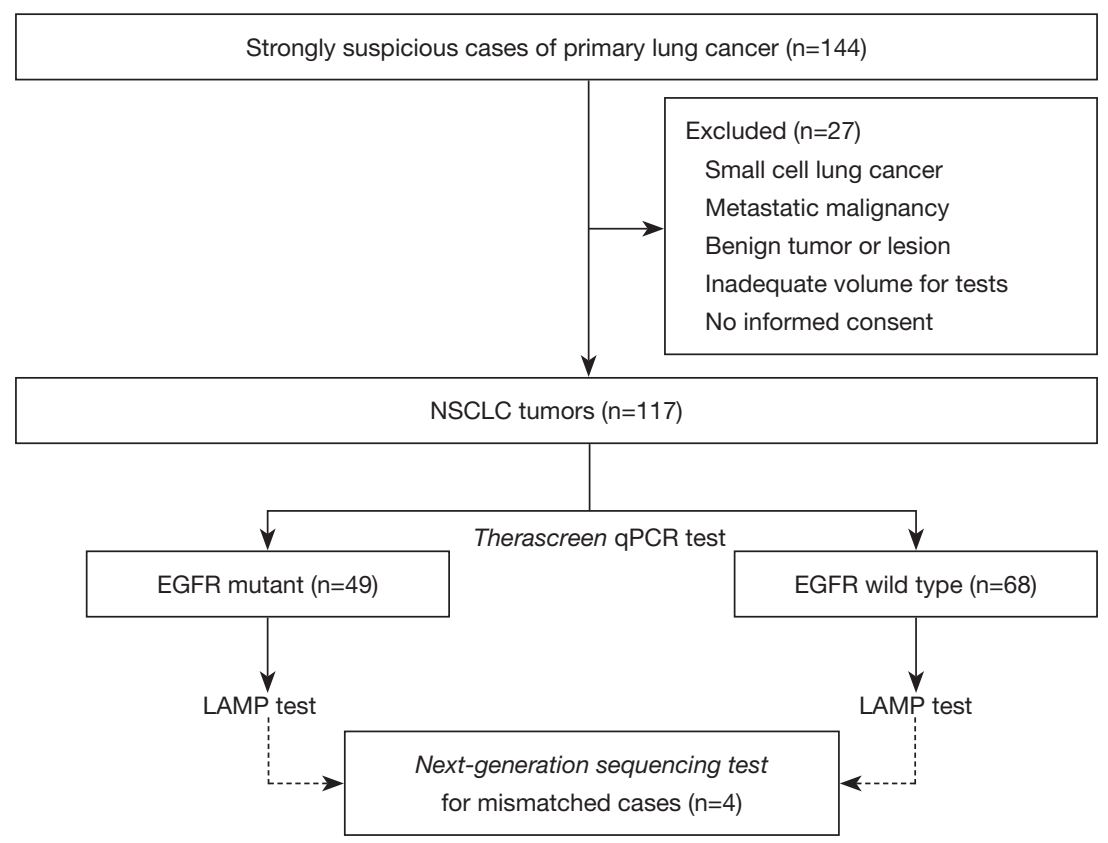

Figure 1 Flowchart of registration. Candidate cases were strongly suspicious primary lung cancer preoperatively. After surgery, all cases without primary non-small cell lung cancer and/or informed consent were excluded from the study. Among candidate cases (n=144), 117 samples were available for testing both therascreen and LAMP assays. Finally, next-generation sequencing was performed for the purpose of investigating EGFR status of discordant cases between therascreen and LAMP assays. NSCLC, non-small cell lung cancer.

Written informed consent was provided by all patients. The study was conducted in accordance with the Declaration of Helsinki (as revised in 2013). There were two primary end points for the study: (I) reproducibility of sensitivity and specificity by using another larger cohort than our previous series, and (II) LAMP AUC value by ROC analysis. All patients with suspicious primary lung cancer underwent surgery or surgical biopsy. After excluding cases of no malignancy or pulmonary metastasis from any other cancer, each specimen was examined microscopically to determine whether an adequate amount of tumor cells was present.

\section{Study population}

We aimed to collect 50 EGFR mutated tumors and over 60 EGFR wild type tumors, since at least one tumor with mismatched EGFR status could be expected between the therascreen and LAMP assays, based on results in our preliminary study.

\section{Tumor tissue samples}

Total 144 samples were obtained for this study from patients with a strong suspicion of primary lung cancer at The Saitama Cardiovascular and Respiratory Center between January 2019 and January 2020 (Figure 1). Among of them, 117 tumor tissues were considered eligible from participants in this study. The mean age of participants was 69.1 years, and they consisted of 77 males and 40 females. Before surgery or biopsy, written informed consent was obtained from consecutive patients with a strong suspicion of primary lung cancer. All specimens were diagnosed by an expert pulmonary pathologist examining HE-stained slides from a low magnification to a high magnification under an ECLIPSE Ni-u light microscope (Nikon Co., Ltd., Tokyo, Japan). Diagnoses were based on the WHO classification version 8 . The inclusion criteria were as follows: (I) NSCLC; (II) enough material volume for molecular testing; and (III) informed written consent from each patient. Cases with small cell lung cancer, pulmonary metastasis from other cancers, absence of informed consent, or insufficient sample volume were excluded. All specimens were fixed with $10 \%$ buffer formalin at $20-25^{\circ} \mathrm{C}(24-36 \mathrm{~h})$ to create formalin-fixed, paraffin-embedded (FFPE) tumor blocks according to the recommendation of The Japanese Society of Pathology (43). Hematoxylin-eosin staining 
was performed by the standard methods using Tissue-Tek Prisma $^{\circledR}$ (Sakura Finetek Japan Co., Ltd., Tokyo, Japan) according to the manufacturer's protocol.

\section{DNA extraction}

To ensure efficient PCR amplification, the tumor content of each sample was assessed by light microscopy at magnifications $\times 10$ to $\times 100$. After sections were deparaffinized with xylene and hydrated through a graded series of ethanol $(100 \%, 100 \%, 85 \%$, and $70 \%$ ethanol), DNA from the tissue blocks was extracted using the QIAampTM DNA FFPE Tissue kit ${ }^{\circledR}$ (Qiagen, Inc., Tokyo, Japan) and analyzed using a QIAcube Robot ${ }^{\circledR}$ (Qiagen, Inc., Tokyo, Japan) according to the manufacturer's protocols (44).

\section{Therascreen qPCR mutation analysis}

The presence of EGFR mutations was determined using a therascreen EGFR PCR kit ${ }^{\circledR}$ (Qiagen, Inc., Tokyo, Japan) according to the manufacturer's protocols (45).

\section{LAMP mutation analysis}

A primer set for targeting EGFR mutation genes was designed using Primer Explorer (primerexplorer.jp/e/) and synthesized by Eurofin Genomics (Eurofins Genomics K.K., Tokyo, Japan). Block oligo and fluorophore-labelled probes were synthesized and purified by Japan Bio Services (Japan Bio Service Co., Ltd., Saitama, Japan) or Gene Design (Gene Design Inc., Osaka, Japan) The LAMP assay was conducted at $65^{\circ} \mathrm{C}$ for 120 minutes by LightCycler $480^{\circledR}$ (Roche Diagnostics K.K.), as described in our previous study (46). After denaturing the amplicon at $95{ }^{\circ} \mathrm{C}$ for $5 \mathrm{~min}$ followed by hybridization at $37^{\circ} \mathrm{C}$ for $5 \mathrm{~min}$, the temperature was gradually raised to $80{ }^{\circ} \mathrm{C}$ and the fluorescent intensity was measured 7 times per $1{ }^{\circ} \mathrm{C}$ increment. The resulting data were analyzed by LightCycler 480 software $^{\circledR}$ (version 1.5.1.62; Roche Diagnostics K.K., Tokyo, Japan) to calculate melting peak.

\section{$N G S$}

NGS was performed for discordant EGFR status cases between therascreen and LAMP assays. Amplicon-based NGS was performed using MiSeq system (Illumina K.K., Tokyo, Japan). The primer sets for amplification of EGFR exons $18,19,20$, and 21 were used as previously reported (46).
The resulting FASTQ files were mapped to GRCh38 human reference sequence by using BWA-MEM (http://bio-bwa.sourceforge.net/). The variant data was extracted from the mapped data by Samtools ver. 1.9 (http:// www.htslib.org/) and GATK4 (https://gatk.broadinstitute. org/hc/en-us).

\section{Statistical analysis}

Descriptive statistics, categorical variables, and the sensitivity and specificity of the LAMP assay were calculated using standard formulae with Excel 2019 ver. 16.0.12527.20260 ${ }^{\circledR}$ (Microsoft Corp., Tokyo, Japan) and SPSS Statistics ${ }^{\circledR}$ version 24 (IBM Corp., Armonk, NY, USA). Standardization of LAMP values and ROC analyses were performed using the statistical software $\mathrm{R}$ version 3.5.1 (R Foundation for Statistical Computing, Vienna, Austria) with ROCR package. Clinical and pathological findings were extracted from the electronic medical records of the patients. To minimize the scattering of LAMP values among primers in the respective locus of EGFR mutations (for example, 18 kinds of primers were used in the LAMP assay to investigate exon 19 deletion), the standardization was performed mathematically to calculate and generate a beeswarm plot of LAMP values. ROC curve analysis was constructed to determine maximum sensitivity and specificity. Under the hypothesis that the EGFR status in the therascreen method would be true, the ROC curve depicted the relationship between true-positive (sensitivity) and false-positive (1 - specificity) cases. The accuracy of the LAMP test was presented by the area under the curve (AUC) and a parametric method based on bivariate normal distribution was used to implement a maximum likelihood estimator for fitting a smooth curve to the data points. The diagnostic AUC values for tumors with and without $E G F R$ mutation were chosen as those values that corresponded to the points on the ROC curves, nearest the upper left hand corner of the graph for optimal balance between sensitivity and specificity. $\mathrm{P}<0.05$ was considered significant.

\section{Results}

\section{Characteristics of patients with NSCLC}

The mean age of the 117 patients was 69.1 years (35-85 years) (Table 1). The majority of patients were male ( $n=77,65.8 \%)$, while 40 patients were female $(34.2 \%)$. In terms of smoking habit, there were 32 never-smokers 
Table 1 Clinical characteristics of study patients

\begin{tabular}{|c|c|}
\hline Characteristic & $N(\%)$ \\
\hline Age, years & $69.1 \pm 9.6$ \\
\hline \multicolumn{2}{|l|}{ Gender } \\
\hline Male & $77(65.8)$ \\
\hline Female & $40(34.2)$ \\
\hline \multicolumn{2}{|l|}{ Smoking Status } \\
\hline Never smoker & $32(27.4)$ \\
\hline Former smoker & $74(63.2)$ \\
\hline Current smoker & $10(8.5)$ \\
\hline \multicolumn{2}{|l|}{ Histology } \\
\hline Adenocarcinoma & $92(78.6)$ \\
\hline Squamous cell carcinoma & $20(17.1)$ \\
\hline Adenosquamous cell carcinoma & $3(2.6)$ \\
\hline Pleomorphic carcinoma & $1(0.9)$ \\
\hline Large cell neuroendocrine carcinoma & $1(0.9)$ \\
\hline \multicolumn{2}{|l|}{ Pathological stage } \\
\hline PO & $1(0.9)$ \\
\hline plA1 & $22(18.8)$ \\
\hline plA2 & $35(29.9)$ \\
\hline plA3 & $13(11.1)$ \\
\hline plB & $17(14.5)$ \\
\hline pllA & $6(5.1)$ \\
\hline pllB & $9(7.7)$ \\
\hline pIIIA & $7(6.0)$ \\
\hline pllIB & $1(0.9)$ \\
\hline plllC & $1(0.9)$ \\
\hline pIVA & $3(2.6)$ \\
\hline pIVB & $2(1.7)$ \\
\hline
\end{tabular}

Data on age: mean \pm SD.

(27.4\%), 74 former-smokers (63.2\%), and 10 current smokers $(8.5 \%)$. Ninety-two cases $(78.6 \%)$ were diagnosed with adenocarcinoma, $20(17.1 \%)$ with squamous cell carcinoma, 3 (2.6\%) with adenosquamous cell carcinoma, $1(0.9 \%)$ with pleomorphic carcinoma, and $1(0.9 \%)$ with large cell neuroendocrine carcinoma. The number of patients in pathological stage 0 , IA1, IA2, IA3, IB, IIA, IIB, IIIA, IIIB, IIIC, IVA, IVB was 1 (0.9\%), 22 (18.8\%),
Table 2 Association between the results from the LAMP assay and the therascreen assay

\begin{tabular}{lcc}
\hline \multirow{2}{*}{ EGFR status } & \multicolumn{2}{c}{ Therascreen PCR } \\
\cline { 2 - 3 } & Positive & Negative \\
\hline LAMP & 47 & 2 \\
Positive & 2 & 66 \\
Negative & & \\
\hline
\end{tabular}

EGFR, epidermal growth factor receptor; LAMP, loop-mediated isothermal amplification.

35 (29.9\%), 13 (11.1\%), 17 (14.5\%), 6 (5.1\%), 9 (7.7\%), $7(6.0 \%), 1(0.9 \%), 1(0.9 \%), 3(2.6 \%)$, and $2(1.7 \%)$, respectively.

\section{Therascreen EGFR PCR mutation analysis}

Among the 117 NSCLCs, there were 49 tumors with EGFR mutations (Table 2), including 25 tumors of exon 19 deletion, 19 tumors of exon $21 \mathrm{~L} 858 \mathrm{R}$ point mutations, 2 tumors of exon 19 deletion/exon 20 in-frame insertion (double mutation), 1 tumor of exon 20 in-frame insertion, and 2 uncommon EGFR mutations (exon 18 G719X point mutation, and double mutation of exon 19 deletion/exon 18 G719X point mutation) (Table 3).

\section{LAMP EGFR mutation analysis}

The LAMP assay detected 49 EGFR mutations (Table 2), however, four mismatched cases were included in therascreen assay. Among the 49 EGFR mutations, there were 26 tumors of exon 19 deletion, 21 tumors of exon 21 L858R point mutations, and 2 uncommon EGFR mutations (exon 18 G719X point mutation, and double mutation of exon $21 \mathrm{~L} 858 \mathrm{R}$ point mutation/exon 18 G719S point mutation) (Table 3).

\section{Comparison of results of therascreen PCR and LAMP assays}

Among the 117 tumors, there were 47 tumors with EGFR mutations and 66 tumors with EGFR wild type in both the therascreen and LAMP assays, i.e., a total of 113 tumors with EGFR mutation status in the LAMP assay were matched with those in the therascreen assay (Table 2). The four tumors that did not match were comprised of two tumors with EGFR mutation in the therascreen assay that were not detected in the LAMP assay, and two tumors with EGFR 
Table 3 EGFR mutation statuses identified using therascreen or LAMP assays

\begin{tabular}{lcc}
\hline Therascreen & LAMP & Number of samples \\
\hline Del 19 & Del 19 & 24 \\
L858R & L858R & 18 \\
G719X & G719X & 1 \\
Del 19 & L858R & 1 \\
Del 19, G719X & Del 19 & 1 \\
Del 19, Ex 20 Ins & L858R & 1 \\
Del 19, Ex 20 Ins & G719S, L858R & 1 \\
Ex 20 Ins & Negative & 1 \\
L858R & Negative & 1 \\
Negative & Del 19 & 1 \\
Negative & L858R & 1 \\
Negative & Negative & 66 \\
\hline
\end{tabular}

EGFR, epidermal growth factor receptor; Exon 18 G719X point mutation, G719X; Exon 19 deletion, Del 19; Exon 20 in-frame insertion, Ex 20 Ins; Exon 21 L858R point mutation, L858R; LAMP, loop-mediated isothermal amplification.

Table 4 Discrepancy between the results from the LAMP assay and the therascreen assay

\begin{tabular}{lccc}
\hline Case number & Therascreen & LAMP & NGS \\
\hline Case 1 & L858R & Negative & L858R \\
Case 2 & Ex 20 Ins & Negative & Negative \\
Case 3 & Negative & Del 19 & Del 19 \\
Case 4 & Negative & L858R & Negative \\
\hline
\end{tabular}

EGFR, epidermal growth factor receptor; Exon 19 deletion, Del 19; Exon 20 in-frame insertion, Ex 20 Ins; Exon 21 L858R point mutation, L858R; NGS, next-generation sequencing; LAMP, loop-mediated isothermal amplification.

mutation in the LAMP assay that were not detected in the therascreen assay. Assuming that the therascreen assay can provide a true EGFR status of NSCLC tumors, sensitivity and specificity of the LAMP assay were $95.9 \%$ and $97.1 \%$, respectively. The positive predictive value and negative predictive value were $95.9 \%$ and $97.1 \%$, respectively.

\section{NGS for four mismatched cases}

NGS was performed to confirm the EGFR mutation disagreement status between the therascreen and the LAMP assays. Among the four cases with mismatched EGFR status, NGS demonstrated concordance with the therascreen assay in two cases (Case 1 and Case 4) and with the LAMP assay in two cases (Case 2 and Case 3) (Table 4).

\section{Receiver operating characteristics (ROC) curve analysis of LAMP EGFR mutations}

For the purpose of further improvement of LAMP assay, ROC curve analysis was performed within tumors with exon 19 deletion or L858R point mutation. The area under the ROC curve (AUC) for LAMP values was 0.973 (95\% CI: $0.929-1.00)$ in exon 19 deletion, and 0.952 (95\% CI: 0.885-1.00) in L858R (Figure 2A,B). Maximized sensitivity, specificity, and accuracy were $89.3 \%, 98.9 \%$, and $96.6 \%$, respectively, based on a cut-off value of 0.806 in exon 19 deletion, and $94.7 \%, 95.9 \%$, and $95.7 \%$, respectively, based on a cut-off value of 0.222 in L858R.

\section{Discussion}

The identification of EGFR mutations has become a standard analysis in the treatment of patients with NSCLC (12-16). However, in spite of the availability of several methods for detecting EGFR mutations (47), to date, there are no point-of-care testing methods that offer simplicity, rapid analysis, and are low-cost. More recently, NGS has been rapidly adopted for use in molecular diagnostic examinations to detect aberrant oncogenes in cancers. This method has the advantage of providing multiple hundreds of genomic loci all at once $(21-25,48)$. However, the technology does not currently meet the demands of the remarkable number of samples, especially in Asian countries, that require testing for lung cancer.

Therascreen EGFR PCR kit $^{\circledR}$ is a US FDA-approved test for the detection of EGFR mutations of lung cancer, and is currently being used in the EU and Japan $(19,20)$. In this study, we compared the performance of the LAMP assay with that of the therascreen assay. Among the 117 tumors tested, 113 tumors (96.6\%) demonstrated the same EGFR status in each assay, while four tumors differed between the two assays (Table 2). The results obtained from the current study were compatible with those from a previous study, which showed sensitivity, specificity, positive predictive value, and negative predictive value of $97.0 \%, 100 \%, 100 \%$, and $96.3 \%$, respectively (46).

NGS was performed to investigate the EGFR mutations 
A

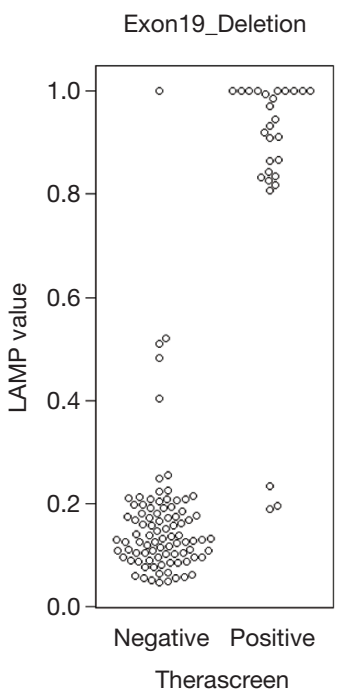

B

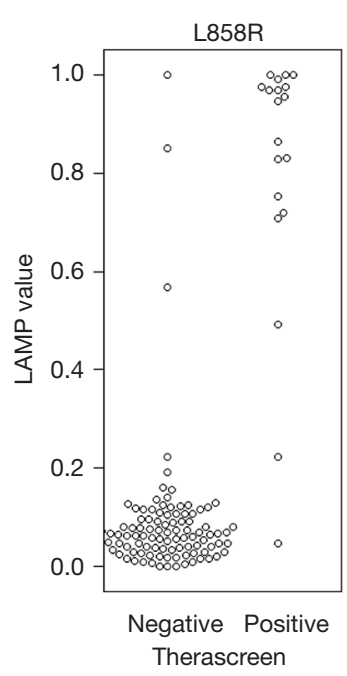

Exon19_Deletion
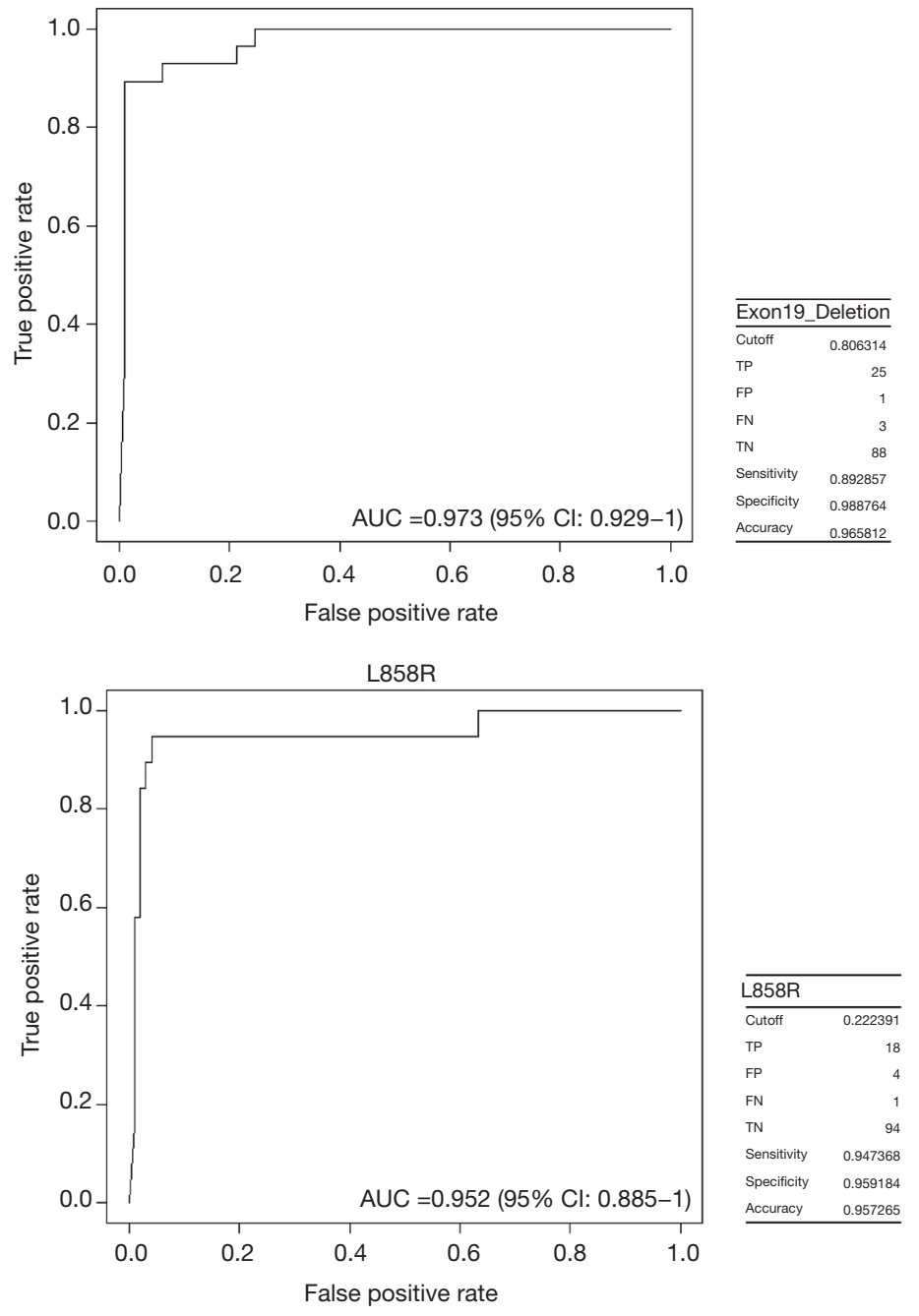

Figure 2 Results and receiver operating characteristics (ROC) curve of the LAMP assay for EGFR mutations. The ROC curve of exon 19 deletion and L858R are shown in Figure $2 A$ and 2B, respectively. All parameters derived from the ROC curve analysis (cut-off value, sensitivity, specificity, and accuracy) are shown in each figure.

of four tumors that had different EGFR statuses between the two assays. Among the four tumors, NGS EGFR status of half of them was in harmony with LAMP EGFR status. Given these results, a conclusion cannot be drawn as to which assay would be more reliable. Therefore, it is important to understand the limitations in of predicting accuracy of oncogenes in clinical settings, and false positives or false negatives should be taken into consideration even when using tests that are recommended by guidelines.

Among the 117 tumors in this study, 51 tumors demonstrated EGFR mutations. In the therascreen assay and/or the LAMP assay, five tumors showed "uncommon" $E G F R$ mutations. Excluding the uncommon EGFR mutations, ROC curves were generated to determine the optimal threshold of LAMP AUC values well distinguish between negative (EGFR wild type) and positive (EGFR mutations) outcomes of the therascreen assay (Figure $2 A, B$ ). The ROC curve of exon 19 deletion gave 0.806 (LAMP value) as a reliable classifier of EGFR status of the therascreen assay, for which high specificity (98.9\%) and high accuracy $(96.6 \%)$ were observed, however the sensitivity (89.3\%) was moderate (Figure $2 A$ ). Conversely, all of the 
parameters (sensitivity, specificity, and accuracy) were extremely satisfactory in L858R when 0.222 was used as a AUC value (Figure 2B). These results could be useful in clinical settings, not only in oncology but also in virology, given the advantages of the LAMP method (rapidity of the test, simplicity of procedure, and versatility of instruments).

In uncommon $E G F R$ mutations, five tumors were detected by the therascreen assay and/or the LAMP assay in this study (Table 3). Among them, only one tumor with G719X was detected by the therascreen assay and the LAMP assay (4/5 cases of uncommon EGFR mutations were discordant between two assays). Although no prospective large-scale trials have evaluated NSCLC tumors with uncommon mutation, and no standard of treatment has been established to date, the sensitivity to EGFR TKIs may significantly vary. For example, high sensitivity to secondgeneration EGFR TKIs in exon 18 mutations and S768I exon 20 point mutation, and high sensitivity to osimertinib and poziotinib in exon 20 insertions have been reported $(49,50)$. Therefore, a study of these rare genetic alterations should be conducted in the future and a more accurate detection system for uncommon EGFR mutations must be developed.

Currently, the therascreen EGFR assay ${ }^{\circledR}$ (Quiagen, Inc.) and the cobas EGFR assay ${ }^{\circledR}$ (Roche Molecular Systems Inc.) for tissue analysis are officially validated by authorized organizations; however, performance of these methods must be improved to meet the global demand in the treatment of various types of EGFR TKIs. Otherwise, effective technological innovations, such as NGS or the LAMP method, must be employed. Despite the limitations of this study (small number of cases and study participants from a single institute), we were able to confirm the feasibility of the LAMP assay for detecting EGFR mutations of NSCLC tumors.

This study had several limitations: (I) the relatively small number of cases evaluated; (II) the single institute nature of the study; and (III) no data of cost-effectiveness. The selling price of the EGFR-LAMP assay is not determined at the moment, however, the TB-LAMP assay can be useful reference for the cost-effectiveness of the LAMP assay (42). In the future, multicentre prospective studies may elucidate the comparison between the LAMP assay and other assay.

In conclusion, our study demonstrates that the LAMP assay is a valuable alternative for detecting EGFR mutations of NSCLC tumors. This is first report indicating an optimal cut-off value that distinguishes EGFR mutations and EGFR wild type in the therascreen assay. In biology or virology, LAMP assay is already rated highly because of reliability, cost-effectiveness, easy handling, and time shortening of turn-around analysis. Our results show that the LAMP assay could be a suitable candidate for point-of-care testing of EGFR mutations in the near future. Now, we are developing a LAMP system for liquid biopsy based on the results from this study (data will be available in our next publication).

\section{Acknowledgments}

The authors would like to thank Dr. Sho Horiuchi, Dr. Hiroaki Morooka, Dr. Takayuki Ibi, and Tomohiko Ikeya for providing explanation for and getting informed consent from patients. We express our gratitude to Mr. Yasuhito Kobayashi and Ms. Mei Miyagawa of the Saitama Cardiovascular and Respiratory Center, Kumagaya, Japan, for their insightful comments and excellent technical assistance.

Funding: The present study was funded by Eiken Chemical Co., Ltd.

\section{Footnote}

Reporting Checklist: The authors have completed the STARD reporting checklist. Available at http://dx.doi.org/10.21037/ jtd-20-2642

Data Sharing Statement: Available at http://dx.doi. org/10.21037/jtd-20-2642

Conflicts of Interest: All authors have completed the ICMJE uniform disclosure form (available at http://dx.doi. org/10.21037/jtd-20-2642). Y Saito, NT, Y Shimizu and EH report grants and non-financial support from Eiken Chemical Co., Ltd., during the conduct of the study. Yukinori Sakao serves as an unpaid editorial board member of Fournal of Thoracic Disease from Sep 2016 to Jul 2021. The other authors have no conflicts of interest to declare.

Ethical Statement: The authors are accountable for all aspects of the work in ensuring that questions related to the accuracy or integrity of any part of the work are appropriately investigated and resolved. The present study was approved by The Institutional Review Board of the Saitama Cardiovascular and Respiratory Center (approval No. 2018032 and 2019038). Written informed consent was provided by all patients. The study was conducted in accordance with the Declaration of Helsinki (as revised in 2013). 
Open Access Statement: This is an Open Access article distributed in accordance with the Creative Commons Attribution-NonCommercial-NoDerivs 4.0 International License (CC BY-NC-ND 4.0), which permits the noncommercial replication and distribution of the article with the strict proviso that no changes or edits are made and the original work is properly cited (including links to both the formal publication through the relevant DOI and the license). See: https://creativecommons.org/licenses/by-nc-nd/4.0/.

\section{References}

1. Ferlay J, Colombet M, Soerjomataram I, et al. Estimating the global cancer incidence and mortality in 2018: GLOBOCAN sources and methods. Int J Cancer 2019;144:1941-53.

2. Siegel RL, Miller KD, Jemal A. Cancer statistics, 2019. CA Cancer J Clin 2019;69:7-34.

3. Lin JJ, Cardarella S, Lydon CA, et al. Five-year survival in EGFR-mutant metastatic lung adenocarcinoma treated with EGFR-TKIs. J Thorac Oncol 2016;11:556-65.

4. Garon EB, Hellmann MD, Rizvi NA, et al. Five-year overall survival for patients with advanced non-small-cell lung cancer treated with pembrolizumab: Results from the phase I KEYNOTE-001 study. J Clin Oncol 2019;37:2518-27.

5. Pacheco JM, Gao D, Smith D, et al. Natural history and factors associated with overall survival in stage IV ALKrearranged non-small cell lung cancer. J Thorac Oncol 2019;14:691-700.

6. Shaw AT, Riely GJ, Bang YJ, et al. Crizotinib in ROS1rearranged advanced non-small-cell lung cancer (NSCLC): updated results, including overall survival, from PROFILE 1001. Ann Oncol 2019;30:1121-6.

7. Singhi EK, Horn L, Sequist LV, et al. Advanced non-small cell lung cancer: Sequencing agents in the EGFR-mutated/ ALK-rearranged populations. Am Soc Clin Oncol Educ Book 2019;39:e187-e197.

8. Zhao D, Chen X, Qin N, et al. The prognostic role of EGFR-TKIs for patients with advanced non-small cell lung cancer. Sci Rep 2017;7:40374.

9. Reck M, Rodríguez-Abreu D, Robinson AG, et al. Updated Analysis of KEYNOTE-024: Pembrolizumab Versus platinum-based chemotherapy for advanced nonsmall-cell lung cancer with PD-L1 tumor proportion score of 50\% or greater. J Clin Oncol 2019;37:537-46.

10. Antonia SJ, Borghaei H, Ramalingam SS, et al. Four-year survival with nivolumab in patients with previously treated advanced non-small-cell lung cancer: a pooled analysis.
Lancet Oncol 2019;20:1395-408.

11. Ettinger DS, Wood DE, Aggarwal C, et al. NCCN Guidelines Insights: Non-Small Cell Lung Cancer, Version 1.2020. J Natl Compr Canc Netw 2019;17:1464-72.

12. Akamatsu H, Ninomiya K, Kenmotsu H, et al. The Japanese Lung Cancer Society Guideline for non-small cell lung cancer, stage IV. Int J Clin Oncol 2019;24:731-70.

13. Kalemkerian GP, Narula N, Kennedy EB, et al. Molecular testing guideline for the selection of patients with lung cancer for treatment with targeted tyrosine kinase inhibitors: American Society of Clinical Oncology Endorsement of the College of American Pathologists/ International Association for the Study of Lung Cancer/ Association for Molecular Pathology Clinical Practice Guideline Update. J Clin Oncol 2018;36:911-9.

14. Ettinger DS, Aisner DL, Wood DE, et al. NCCN guidelines®insights non-small cell lung cancer, version 5.2018 featured updates to the NCCN guidelines. J Natl Compr Cancer Netw 2018;16:807-21.

15. Postmus PE, Kerr KM, Oudkerk M, et al. Early and locally advanced non-small-cell lung cancer (NSCLC): ESMO Clinical Practice Guidelines for diagnosis, treatment and follow-up. Ann Oncol 2017;28:iv1-iv21.

16. The Japanese Lung Cancer Society: Guidelines of Lung Cancer. Available online: https//www.haigan.gr.jp/ modules/guideline/index.php?content_id=3 (accessed 7 August 2020).

17. Maemondo M, Inoue A, Kobayashi K, et al. Gefitinib or chemotherapy for non-small-cell lung cancer with mutated EGFR. N Engl J Med 2010;362:2380-8.

18. The Japan Lung Cancer Society: Guidelines for Gefitinib Use. Available online: https//www.mhlw.go.jp/ shingi/2007/02/dl/s0201-4c.pdf (accessed 7 August 2020)

19. Kazandjian D, Blumenthal GM, Yuan W, et al. FDA Approval of Gefitinib for the Treatment of Patients with Metastatic EGFR Mutation-Positive Non-Small Cell Lung Cancer. Clin Cancer Res 2016;22:1307-12.

20. The Japan Lung Cancer Society, Guidance of examinations of EGFR mutations for patients with Lung Cancer ver4.0. Available online: https//www.haigan.gr.jp/modules/ guideline/index.php?content_id=7 (accessed 7 August 2020).

21. Tan AC, Lai GGY, Tan GS, et al. Utility of incorporating next-generation sequencing (NGS) in an Asian non-small cell lung cancer (NSCLC) population: Incremental yield of actionable alterations and cost-effectiveness analysis. Lung Cancer 2020;139: 207-15.

22. Kashima J, Kitadai R, Okuma Y. Molecular and 
morphological profiling of lung cancer. A foundation for "next-generation" pathologists and oncologists. Cancers (Basel) 2019;11:599.

23. Takeda M, Sakai K, Takahama T, et al. New era for next-generation sequencing in Japan. Cancers (Basel) 2019;11:742.

24. Legras A, Barritault M, Tallet A, et al. Validity of targeted next-generation sequencing in routine care for identifying clinically relevant molecular profiles in non-small-cell lung cancer: Results of a 2-year experience on 1343 samples. J Mol Diagn 2018;20:550-64.

25. Takeda M, Sakai K, Terashima M, et al. Clinical application of amplicon-based next-generation sequencing to therapeutic decision making in lung cancer. Ann Oncol 2015;26:2477-82.

26. Notomi T, Okayama H, Masubuchi H, et al. Loopmediated isothermal amplification of DNA. Nucleic Acids Res 2000;28:E63.

27. Notomi T, Mori Y, Tomita N, et al. Loop-mediated isothermal amplification (LAMP): principle, features, and future prospects. J Microbiol 2015;53:1-5.

28. Zhang H, Xu Y, Fohlerova Z, et al. LAMP-on-a-chip: Revising microfluidic platforms for loop-mediated DNA amplification. Trends Analyt Chem 2019;113:44-53.

29. Takano C, Kuramochi Y, Seki M, et al. Molecular serotype-specific identification of Streptococcus pneumoniae using loop-mediated isothermal amplification. Sci Rep 2019;9:19823.

30. Quyen TL, Nordentoft S, Vinayaka AC, et al. Specific and Simple Loop Mediated Isothermal Amplification Method for Rapid Detection of Campylobacter Spp. In Broiler Production. Front Microbiol 2019;10:2443.

31. Wang Y, Wang Y, Jiao W, et al. Development of loopmediated isothermal amplification coupled with nanoparticle-based lateral flow biosensor assay for Mycoplasma pneumoniae detection. AMB Express 2019;9:196.

32. Nagai K, Horita N, Yamamoto M, et al. Diagnostic test accuracy of loop-mediated isothermal amplification assay for Mycobacterium tuberculosis: systematic review and meta-analysis. Sci Rep 2016;6:39090.

33. Mallepaddi PC, Lai MY, Podha S, et al. Development of loop-mediated isothermal amplification-based lateral flow device method for the detection of malaria. Am J Trop Med Hyg 2018;99:704-8.

34. King KM, Hawkins NJ, Atkins S, et al. First application of loop-mediated isothermal amplification (LAMP) assays for rapid identification of mating type in the heterothallic fungus Aspergillus fumigatus. Mycoses 2019;62:812-7.

35. Ihira M, Yoshikawa T, Enomoto Y, et al. Rapid diagnosis of human herpesvirus 6 infection by a novel DNA amplification method, loop-mediated isothermal amplification. J Clin Microbiol 2004;42:140-5.

36. Jin Y, Yang H, Ji W, et al. Virology, epidemiology, pathogenesis, and control of COVID-19. Viruses 2020;12:372.

37. Coronaviridae Study Group of the International Committee on Taxonomy of Viruses. The species Severe acute respiratory syndrome-related coronavirus: classifying 2019-nCoV and naming it SARS-CoV-2. Nat Microbiol 2020;5:536-44.

38. Bustin SA, Nolan T. RT-qPCR Testing of SARS-CoV-2: A Primer. Int J Mol Sci 2020;21:3004.

39. Corman VM, Landt O, Kaiser M, et al. Detection of 2019 novel coronavirus (2019-nCoV) by real-time RT-PCR. Euro Surveill 2020;25:2000045.

40. Lu R, Wu X, Wan Z, et al. Development of a novel reverse transcription loop-mediated isothermal amplification method for rapid detection of SARS-CoV-2. Virol Sin 2020;35:344-7.

41. Park GS, Ku K, Baek SH, et al. Development of reverse transcription loop-mediated isothermal amplification assays targeting severe acute respiratory syndrome coronavirus 2 (SARS-CoV-2). J Mol Diagn 2020;22:729-35.

42. World Health Organization, The use of loop-mediated isothermal amplification (TB-LAMP) for the diagnosis of pulmonary tuberculosis: policy guidance. Available online: https://www.who.int/tb/publications/lamp-diagnosismolecular/en/ (accessed 4 November 2020).

43. The Japanese Society of Pathology, Regulations for handling pathological tissue specimens for genomic medicine. Available online: http://pathology.or.jp/genome_ med/pdf/textbook.pdf (accessed 7 August 2020).

44. Angulo B, García-García E, Martínez R, et al. A commercial real-time PCR kit provides greater sensitivity than direct sequencing to detect KRAS mutations: a morphology-based approach in colorectal carcinoma. J Mol Diagn 2010;12:292-9.

45. Vallée A, Le Loupp AG, Denis MG. Efficiency of the Therascreen® RGQ PCR kit for the detection of EGFR mutations in non-small cell lung carcinomas. Clin Chim Acta 2014;429:8-11.

46. Horiuchi S, Saito Y, Matsui A, et al. A novel loopmediated isothermal amplification method for efficient and robust detection of EGFR mutations. Int J Oncol 2020;56:743-9. 
47. Pinsolle J, McLeer-Florin A, Giaj Levra M, et al. Translating systems medicine into clinical practice: Examples From pulmonary medicine with genetic disorders, infections, inflammations, cancer genesis, and treatment implication of molecular alterations in nonsmall-cell lung cancers and personalized medicine. Front Med (Lausanne) 2019;6:233.

48. The Japanese Lung Cancer Society, Guidance of genetic screening by next-generation Sequencing. Available online: https//www.haigan.gr.jp/modules/guideline/index. php? content_id=40 (accessed 7 August 2020).

Cite this article as: Saito $\mathrm{Y}$, Takahashi N, Matsui A, Michiyuki S, Yamauchi Y, Shimizu Y, Hoshi E, Sakao Y, Kawamura M. Comparative study of the loop-mediated isothermal amplification method and the QIAGEN therascreen PCR kit for the detection of EGFR mutations in non-small cell lung cancer. J Thorac Dis 2021;13(2):743-753. doi: 10.21037/ jtd-20-2642
49. Russo A, Franchina T, Ricciardi G, et al. Heterogeneous responses to epidermal growth factor receptor (EGFR) tyrosine kinase inhibitors (TKIs) in patients with uncommon EGFR mutations: New insights and future perspectives in this complex clinical scenario. Int J Mol Sci 2019;20:1431.

50. Gristina V, Malapelle U, Galvano A, et al. The significance of epidermal growth factor receptor uncommon mutations in non-small cell lung cancer: A systematic review and critical appraisal. Cancer Treat Rev 2020;85:101994. 